\title{
Formation and development of shock waves in the solar corona and the near-Sun interplanetary space
}

\author{
G. Mann, A. Klassen, H. Aurass, and H.-T. Classen \\ Astrophysikalisches Institut Potsdam, An der Sternwarte 16, 14482 Potsdam, Germany \\ Received 24 January 2002 / Accepted 16 September 2002

\begin{abstract}
At the Sun, shock waves are produced either by flares and/or by coronal mass ejections (CMEs) and are regarded as the source of solar energetic particle events. They can be able to generate solar type II radio bursts. The non-radial propagation of a disturbance is considered away from an active region through the corona into the interplanetary space by evaluating the spatial behaviour of the Alfvén speed. The magnetic field of an active region is modelled by a magnetic dipole superimposed on that of the quiet Sun. Such a magnetic field structure leads to a local minimum of the Alfvén speed in the range 1.2-1.8 solar radii in the corona as well as a maximum of $740 \mathrm{~km} \mathrm{~s}^{-1}$ at a distance of 3.8 solar radii. The occurrence of such local extrema has important consequences for the formation and development of shock waves in the corona and the near-Sun interplanetary space and their ability to accelerate particles. It leads to a temporal delay of the onset of solar energetic particle events with respect to both the initial energy release (flare) and the onset of the solar type II radio burst.
\end{abstract}

Key words. Sun: flares - Sun: coronal mass ejections (CMEs) - Sun: magnetic field - Sun: particle emission Sun: radio radiation - shock waves

\section{Introduction}

In the solar corona, shock waves can occur as blast waves due to the flare process (Uchida et al. 1973; Vršnak et al. 1995) or shocks driven by coronal mass ejections (CMEs) (Stewart et al. 1974a,b). Some of them can continue as travelling shocks in the interplanetary space. Coronal and interplanetary shocks can be the source of type II radio radiation in the meteric (Wild \& McCready 1950; Uchida 1960) and deca-hectometric wave range (Cane et al. 1981; Reiner \& Kaiser 1999), respectively. Type II radio bursts appear as stripes of enhanced radio emission slowly drifting from high to low frequencies in dynamic radio spectra (see Nelson \& Melrose 1985; Mann 1995; and Aurass 1996 for reviews). A particular example is presented in Fig. 1. It was recorded by the radio spectralpolarimeter (Mann et al. 1992) of the Astrophysikalisches Institut Potsdam on November 18, 2000. The solar type II radio burst started at $130 \mathrm{MHz}$ and $260 \mathrm{MHz}$ on 13:11:42 UT with two emission stripes slowly drifting towards lower frequencies. These stripes are usually called a "backbone" and are considered as the signature of the associated shock wave travelling outwards in the corona (Nelson \& Melrose 1985). The fundamentalharmonic structure of the "backbone" is evidently seen in this particular event. Later on, several emission stripes shooting up from the "backbone" towards lower frequencies appeared after 13:14:30 UT. They represent the so-called "shock-associated" (SA) type III bursts (Cane 1981). Usually type III radio bursts

Send offprint requests to: G. Mann, e-mail: GMann@aip.de are interpreted as the radio signature of electron beams propagating along open magnetic field lines in the corona and, partly, into the interplanetary space (Suzuki \& Dulk 1985). Therefore, the SA type III bursts are considered as beams of electrons which are generated by the shock wave associated with the "backbone" (Nelson \& Melrose 1985).

Cane (1983) suggested the appearance of two different kinds of shocks, the flare produced blast waves associated with the solar type II radio bursts in the inner corona (Wagner \& MacQueen 1983) and CME driven interplanetary shocks, which are considered to be the source of long-living solar energetic particle events (SEPs) (Kahler 1994; Reames et al. 1996). Recently, Gopalswamy et al. (1998) investigated a large sample of type II bursts including also the low frequency range $(\leq 14 \mathrm{MHz})$ covered by the radio spectrometer WAVES (Bougeret et al. 1995) aboard the Wind spacecraft. "The result of the study is that the flare-produced coronal and the transient interplanetary shocks produced by CMEs are independent of each other." (Gopalswamy et al. 1998). A similar result has recently been presented by Classen \& Aurass (2002). Both studies confirm the suggestion by Cane (1983).

Yohkoh and SOHO images of the Sun and complementary spatial radio-heliographic observations revealed that solar type II radio burst sources mainly propagate non-radially away from active regions (Gopalswamy et al. 1997, 2000; Aurass et al. 1998; Klassen et al. 1999; Klein et al. 1999). Type II burst "sources ... are non-radial displaced from the axis of the active region loops" (Klassen et al. 1999), "but seems to be related to the orientation of ... the motion of the plasma blob" 


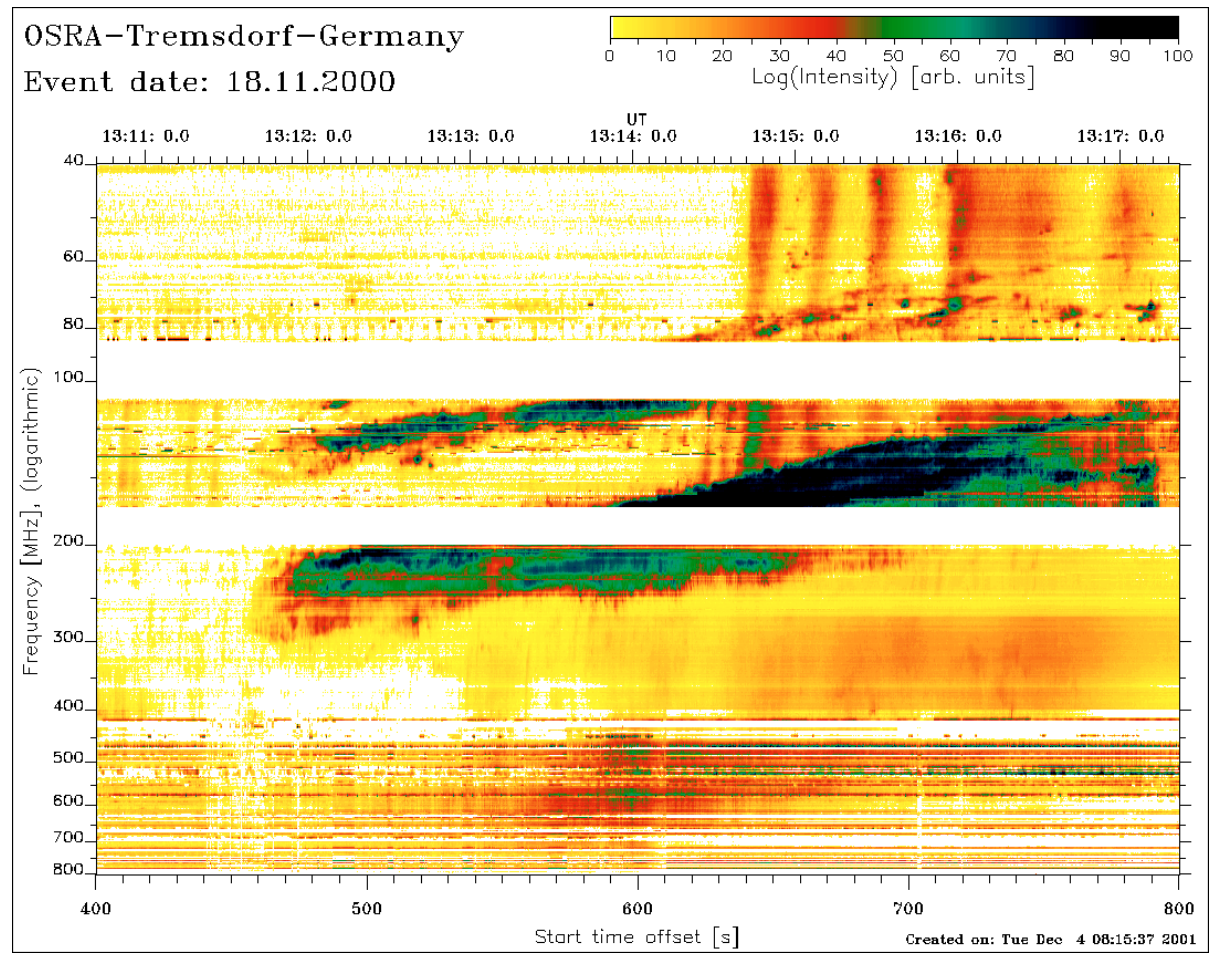

Fig. 1. Solar type II radio burst during the solar event on November 18, 2000.

(Klein et al. 1999) associated with the shock. In order to study the formation and development of shock waves in the solar corona and the interplanetary space, it is necessary to know the behaviour of the Alfvén speed along the propagation path of the disturbance associated with the shock wave. That requires a model of the magnetic field of an active region superimposed on that of the quiet Sun and a density model (Sect. 2). The formation and development of a shock wave in the corona and the interplanetary space is considered in Sect. 3. It is carried out much more in detail as previously done by Mann et al. (1999a). In contrast to Gopalswamy et al. (2001), who only adopted a purely radial magnetic field model (Dulk \& McLean 1978), a non-radial magnetic field model in terms of a magnetic dipole is used in the presented paper (Sect. 2). Such an approach is more appropriate to study the non-radial propagation of a shock wave as that by Gopalswamy et al. (2001).

It is emphasized that the values of the various parameters adopted in the paper can vary from case to case in reality. They are used in the discussion in order to determine the possible spatial and temporal scales of the formation and development of shock waves in a quantitatively straightforward manner. The results should be regarded in such a context in this paper. The results of this study, especially those concerning SEPs, are discussed in Sect. 4.

\section{The model}

In order to study the formation and development of shock waves, the behaviour of the Alfvén speed as the characteristic speed in a magnetoplasma should be investigated in the solar corona and the interplanetary space. Since the Alfvén speed depends on the magnitude $B$ of the magnetic field and the full particle number density $N$, a model of the magnetic field of an active region superimposed on that of the quiet Sun and a density model is needed.

In the solar corona, shock waves are established near but out of active regions (Aurass et al. 1998; Klassen et al. 1999; Klein et al. 1999; Gopalswamy et al. 2000). Therefore, a onefold Newkirk (1961) model

$N_{\mathrm{e}}=N_{0} \times 10^{4.32 R_{\mathrm{S}} / R}$

$\left(N_{0}=4.2 \times 10^{4} \mathrm{~cm}^{-3} ; R_{\mathrm{S}}\right.$, radius of the Sun $)$ is chosen as an appropriate model of the electron number density $N_{\mathrm{e}}$ for this study. The white-light scattering observations of the corona by Koutchmy (1994) showed that the one-fold Newkirk (1961) model fits the conditions well above quiet equatorial regions. Here and henceforth, $R$ denotes the radial distance from the center of the Sun. Note that the Newkirk (1961) model corresponds to a barometric height formula with a temperature of $1.4 \times 10^{6} \mathrm{~K}$. For $R / R_{\mathrm{S}} \rightarrow \infty$ the Newkirk model provides an electron number density of $4.2 \times 10^{4} \mathrm{~cm}^{-3}$. However, a typical value of about $6 \mathrm{~cm}^{-3}$ is measured at $1 \mathrm{AU}$ (Schwenn 1990). The reason for this discrepancy is the solar wind, while the Newkirk model is a hydrostatic one. Mann et al. (1999b) presented a heliospheric density model as a special solution of Parker's (1958) wind equation. It agrees very well with observations made from the corona up to $5 \mathrm{AU}$. It provides an electron number density of $5.14 \times 10^{9} \mathrm{~cm}^{-3}$ at the bottom of the corona. According to the observations by Koutchmy (1994) such values are usually found above active regions. Since a density model above quiet equatorial regions is needed for this study, the one-fold Newkirk (1961) model is used for the regions $R \leq 1.8 R_{\mathrm{S}}$, whereas that by Mann et al. (1999b) is adopted in the region $R \geq 1.8 R_{\mathrm{S}}$ (Fig. 2). Leblanc et al. (1998) 


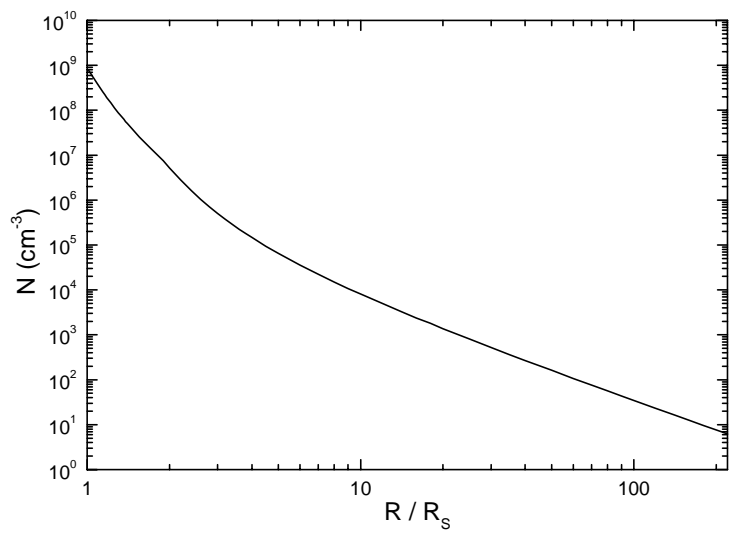

Fig. 2. Radial behaviour of the electron number density according to the one-fold Newkirk (1961) for the solar corona and the heliospheric density model by Mann et al. (1999b) for the interplanetary space.

traced the electron number density from the corona up to $1 \mathrm{AU}$ by evaluating Wind data. Their study confirms the model by Mann et al. (1999b). The electron number density $N_{\mathrm{e}}$ is related to the full particle number density $N$ by $N=1.92 N_{\mathrm{e}}$ for a mean molecular weight $\mu=0.6$, which is an appropriate value in the solar corona (Priest 1982).

As already mentioned, the complete magnetic field $\boldsymbol{B}$ can be composed of that of an active region $\boldsymbol{B}_{\text {ar }}$ and of the quiet Sun $\boldsymbol{B}_{\mathrm{qS}}$, i.e.

$\boldsymbol{B}=\boldsymbol{B}_{\mathrm{ar}}+\boldsymbol{B}_{\mathrm{q} S}$

Here, an active region is modelled by a magnetic dipole with the moment $\boldsymbol{M}$ and a length $\lambda . \lambda=0.1 R_{\mathrm{S}}$ is adopted because of the assumed width of the hydrogen convection zone. It is located in a depth $\lambda / 2$ under the photosphere. The magnetic field of such a dipole is given by

$\boldsymbol{B}_{\mathrm{ar}}=\frac{3(\boldsymbol{M} \cdot \boldsymbol{r}) \boldsymbol{r}}{r^{5}}-\frac{\boldsymbol{M}}{r^{3}}$

(Landau \& Lifshitz 1975), where $r$ denotes the distance from the center of the dipole. Cylindrical coordinates are introduced in such a way that the magnetic dipole is directed along the $z$-axis and azimuthal symmetry is assumed with respect to the $z$-axis. Then, a point $P$ is unambiguously characterized either by its distance $\rho$ from the $z$-axis and its radius $R$ from the center of the Sun or by the distance $r$ from the center of the dipole and the angle $\gamma$ (Fig. 3). In this frame of reference the magnetic field (see Eq. (3)) is given by

$$
\begin{aligned}
& B_{\mathrm{ac}, z}(r)=B_{0} \cdot \frac{\lambda^{3}}{16} \cdot\left(\frac{R_{\mathrm{S}}}{r}\right)^{3} \cdot\left(3 \cos ^{2} \gamma-1\right) \\
& B_{\mathrm{ac}, \rho}(r)=B_{0} \cdot \frac{\lambda^{3}}{16} \cdot\left(\frac{R_{\mathrm{S}}}{r}\right)^{3} \cdot(3 \cos \gamma \sin \gamma)
\end{aligned}
$$

where $B_{0}$ is defined as the magnitude of the magnetic field on the axis of the dipole (i.e. $\gamma=0^{\circ}$ ) at a distance $r=\lambda / 2$ (i.e. at the photosphere). Consequently, the magnetic moment ( $\boldsymbol{M}=$ $M \cdot \boldsymbol{e}_{z} ; \boldsymbol{e}_{z}$, unit vector along the $z$-axis) has a value $M=B_{0} \lambda^{3} / 16$. Here, $B_{0}=0.8 \mathrm{kG}$ is taken as a typical value of the magnetic field in an active region (see Priest 1982).

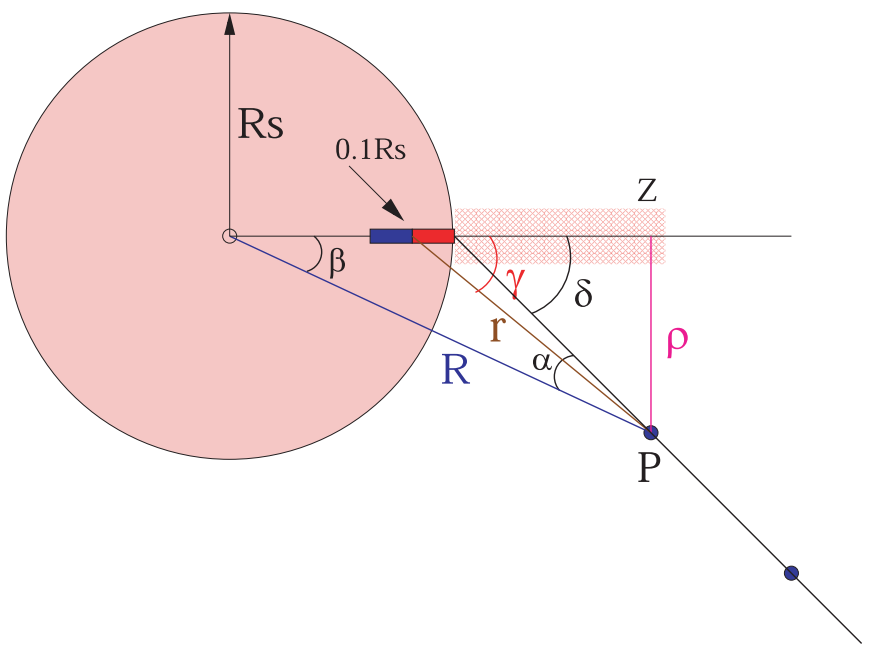

Fig. 3. Scheme of reference frame used in the paper.

In the same frame of reference the magnetic field of the quiet Sun is given by

$$
\begin{gathered}
B_{\mathrm{qS}, z}=B_{\mathrm{S}} \cdot \cos \beta \cdot\left(\frac{R_{\mathrm{S}}}{R}\right)^{2} \\
B_{\mathrm{qS}, \rho}=B_{\mathrm{S}} \cdot \sin \beta \cdot\left(\frac{R_{\mathrm{S}}}{R}\right)^{2}
\end{gathered}
$$

with $\beta=\arcsin (\rho / R)$ (Fig. 3). The radial behaviour of the magnitude

$B_{\mathrm{qS}}(R)=B_{\mathrm{S}} \cdot\left(\frac{R_{\mathrm{S}}}{R}\right)^{2}$

(with $B_{\mathrm{S}}=2.2 \mathrm{G}$ ) of the magnetic field of the quiet Sun has been adopted from Mann et al. (1999a). It was obtained from the analysis of the propagation of coronal transient (or EIT) waves (Klassen et al. 2000) by assuming the conservation of the magnetic flux and neglecting the azimuthal component of the magnetic field with respect to the radial one (Mann et al. 1999a). Note that $2.2 \mathrm{G}$ is a typical value of the magnetic field in quiet photospheric regions (see Priest 1982). Equation (8) provides a magnetic field of $4.8 \mathrm{nT}$ at $1 \mathrm{AU}$. Similar values are acutally measured by spacecraft (Mariani \& Neubauer 1990). The behaviour of the magnitude of the magnetic field as given in Eq. (8) roughly agrees with the solar magnetic field model by Banaszkiewicz et al. (1998), especially near the ecliptic plane, i.e. above quiet equatorial regions.

The magnetic fields of the dipole and the quiet Sun can be superimposed in two different manners: Along the $z$-axis both magnetic fields are directed either parallel ("+" sign) or antiparallel ("-" sign). Henceforth, these cases are called the "parallel" and/or "anti-parallel" case. Then, the composed magnetic field is obtained by vector addition (Eq. (2)). The magnitude $B=|\boldsymbol{B}|$ of $\boldsymbol{B}$ is finally entering into the calculations of the Alfvén speed.

In order to study the formation and development of shock waves in the corona and the interplanetary space, the propagation of a disturbance along a straight path away from the center of an active region (i.e. $z=R_{\mathrm{S}}$ and $\beta=0^{\circ}$ ) into the interplanetary space is considered. This straight line takes an angle $\delta$ with 
respect to the $z$-axis (Fig. 3). Then, for an arbitrary point $P$ on this line, $\rho$ is related to $R$ by

$\frac{\rho}{R_{\mathrm{S}}}=\sin \delta \cdot\left[\sqrt{\frac{R^{2}}{R_{\mathrm{S}}^{2}}-\sin ^{2} \delta}-\cos \delta\right]$

and, consequently, the angle $\vartheta$ (Fig. 3) can be calculated by

$\vartheta=\arctan \left[\frac{1}{\cot \delta+\frac{\lambda}{2} \cdot \frac{R_{\mathrm{S}}}{R}}\right]$

leading to the determination of the radial distance $r$

$\frac{r}{R_{\mathrm{S}}}=\frac{\rho}{R_{\mathrm{S}}} \cdot \frac{1}{\sin \vartheta}$

from the center of the magnetic dipole. Finally, the angle $\alpha$ between the straight line and the direction radially away from the Sun is given by

$\alpha=\delta-\beta$

(Fig. 3). Now, the magnetic field (Eqs. (4)-(7)) can be calculated at each point $P$ along the straight line for a given value of $\delta$ by using Eqs. (9)-(11). Note that each point $P$ is unambiguously defined by its radial distance $R$ from the center of the Sun for a given value of $\delta$. Thus, the electron number density can be found at this point $P$ according to the density model (Fig. 2) already introduced in this section.

The behaviour of the local Alfvén speed along such straight lines can be evaluated in the parallel ( $v_{\mathrm{A}}^{+}$; full line) and anti-parallel $\left(v_{\mathrm{A}}^{-}\right.$; dashed line) case. This is shown for $\delta=$ $15^{\circ}, 45^{\circ}, 75^{\circ}$ in Fig. 4. Since the one-fold Newkirk (1961) model is only appropriate outside active regions, the Alfvén speeds presented in Fig. 4 are only meaningful for radial distances that are sufficiently far from the active region. Active regions have a typical diameter of $2.4^{\prime}=105000 \mathrm{~km}=0.15 R_{\mathrm{S}}$ (Priest 1982). Thus, $\rho$ (Fig. 3) should be greater than $0.08 R_{\mathrm{S}}$ in order to be sufficiently far from the active region. It is the case for radial distances beyond $1.301 R_{\mathrm{S}}, 1.083 R_{\mathrm{S}}$, and $1.025 R_{\mathrm{S}}$ for $\delta=15^{\circ}, 45^{\circ}$, and $75^{\circ}$, respectively. The dependence of the Alfvén speed from the angle $\delta$ on a fixed radial level at $1.32 R_{\mathrm{S}}$ is presented in Fig. 5. According to the density model used in this paper (Fig. 2), an electron number density of $7.87 \times 10^{7} \mathrm{~cm}^{-3}$ corresponding to a plasma frequency of $80 \mathrm{MHz}$ is related to this level. Since a fast magnetosonic wave is refracted towards regions of low Alfvén speeds, they are refracted away from and towards an active region in the parallel and anti-parallel case, respectively (Fig. 5).

The influence of the magnetic field of an active region modelled by a magnetic dipole becomes negligible beyond a distance of $2.2 R_{\mathrm{S}}$, i.e. the magnetic field of the quiet Sun dominates there (see also Mann et al. 1999a; and Gopalswamy et al. 2001). In order to illustrate the behaviour of the Alfvén speed from the corona up to the interplanetary space (i.e. up to $\left.1 \mathrm{AU}\left(=214 R_{\mathrm{S}}\right)\right)$ in a representative manner, $\delta=45^{\circ}$ is chosen in Fig. 6. The dotted line shows the Alfvén speed resulting only from the magnetic field of the quiet Sun (i.e. according to Eq. (8)) (see also Mann et al. 1999a).

On the one hand, the Alfvén speed has a local maximum of $740 \mathrm{~km} \mathrm{~s}^{-1}$ at $3.8 R_{\mathrm{S}}$ as revealed by inspecting Fig. 6 (see

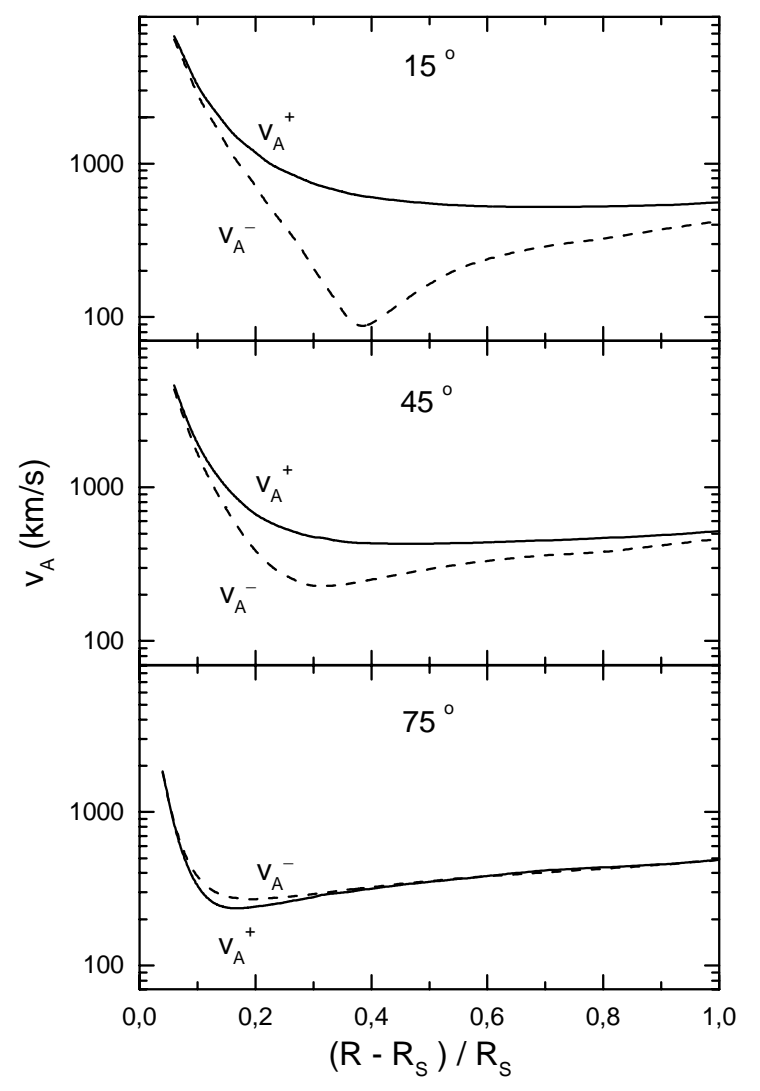

Fig. 4. Alfvén speed along a straight line away from an active region as a function of the radial distance $R$ for three different values of $\delta$ ( $\delta=$ $\left.15^{\circ}, 45^{\circ}, 75^{\circ}\right)$. The full and dashed lines indicate the Alfvén speeds in the parallel $\left(v_{\mathrm{A}}^{+}\right)$and anti-parallel $\left(v_{\mathrm{A}}^{-}\right)$case, respectively.

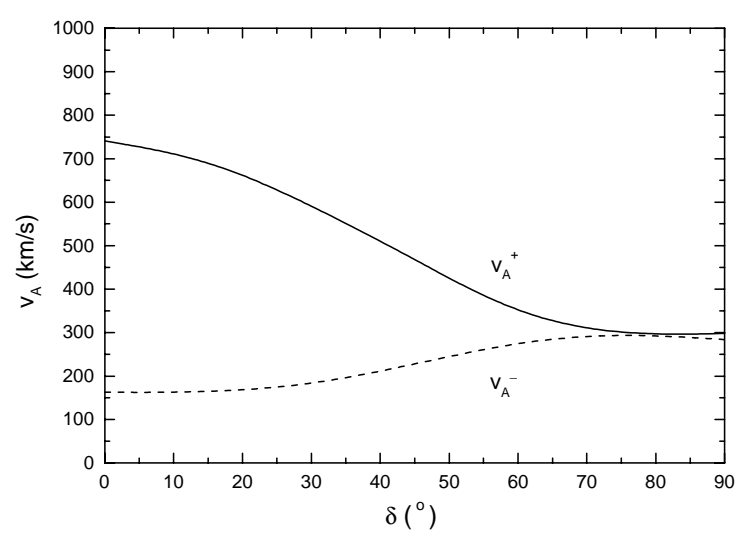

Fig. 5. Dependence of the Alfvén speeds $v_{\mathrm{A}}^{+}$and $v_{\mathrm{A}}^{-}$on the angle $\delta$ for a fixed radial distance $R=1.32 R_{\mathrm{S}}$.

also Mann et al. 1999a; Gopalswamy et al. 2001). On the other hand, the Alfvén speed has a local minimum in the corona below $2 R_{\mathrm{S}}$ (see also Gopalswamy et al. 2001). The location of this minimum is strongly dependent on the angle $\delta$ as shown in Fig. 4. In the parallel case the minima of the Alfvén speeds have values of $519 \mathrm{~km} \mathrm{~s}^{-1}, 428 \mathrm{~km} \mathrm{~s}^{-1}$, and $236 \mathrm{~km} \mathrm{~s}^{-1}$ at $1.70 R_{\mathrm{S}}$, $1.45 R_{\mathrm{S}}$, and $1.16 R_{\mathrm{S}}$ for $\delta=15^{\circ}, 45^{\circ}$, and $45^{\circ}$, respectively (Fig. 4). Furthermore, the Alfvén speed takes a minimum of $86 \mathrm{~km} \mathrm{~s}^{-1}, 227 \mathrm{~km} \mathrm{~s}^{-1}$, and $271 \mathrm{~km} \mathrm{~s}^{-1}$ at $1.38 R_{\mathrm{S}}, 1.32 R_{\mathrm{S}}$, and $1.19 R_{\mathrm{S}}$ for $\delta=15^{\circ}, 45^{\circ}$, and $75^{\circ}$ in the anti-parallel case, 


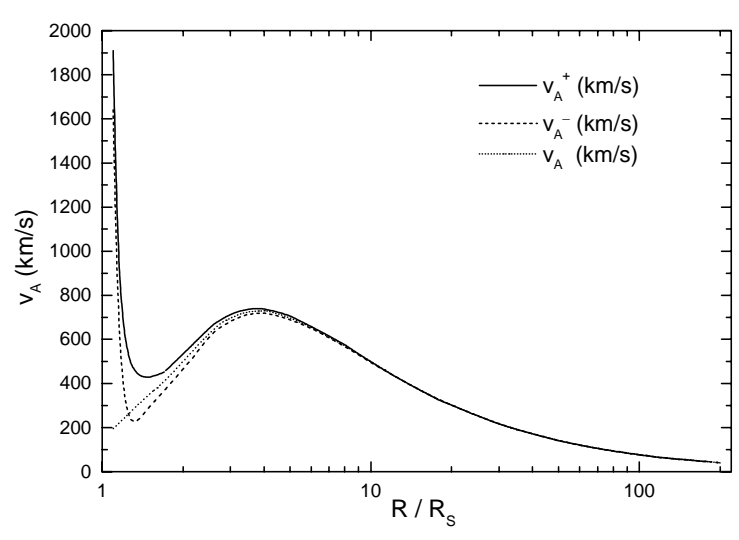

Fig. 6. Alfvén speed along a straight line with $\delta=45^{\circ}$ away from an active region as a function of the radial distance $R$. The full and dashed lines indicate the Alfvén speeds in the parallel $\left(v_{\mathrm{A}}^{+}\right)$and anti-parallel $\left(v_{\mathrm{A}}^{-}\right)$case, respectively. The dotted line represents the behaviour of the Alfvén speed only due to the magnetic field of the quiet Sun.

respectively (Fig. 4). In this case the minimum of the Alfvén speed has relatively low values, which are comparable with the sound speed $\left(180 \mathrm{~km} \mathrm{~s}^{-1}\right)$.

Recently, Gopalswamy et al. (2001) discussed the radial behaviour of the fast magnetosonic speed from the solar corona up to the interplanetary space. They chose the model by Dulk $\&$ McLean (1978) for the magnetic field above active regions. This model gives only the radial behaviour of the magnitude of the magnetic field and provides a value of $500 \mathrm{G}$ at a distance of $1.01 R_{\mathrm{S}}$. The magnetic dipole as a model of an active region additionally allows one to consider the lateral behaviour of the magnetic field in contrast to the model by Dulk $\&$ McLean (1978). The magnetic field model used in their paper provides an magnetic field strength of $436 \mathrm{G}$ at a distance of $1.01 R_{\mathrm{S}}$. Thus, both models are similar to each other along the axis of the dipole, i.e. above the active regions. But, because coronal shocks such as type II burst sources mainly propagate nonradially away from active regions, the magnetic field model adopted in this paper is more appropriate for studying the formation and development of shock waves in the solar corona than the approach used by Gopalswamy et al. (2001). Furthermore, the magnetic field of the active region (in terms of a magnetic dipole) and that of the quiet Sun are regarded as vector quantities, whereas Gopalswamy et al. (2001) considered only the magnitude of the magnetic field. Therefore, the regions of relatively low Alfvén speeds (see Fig. 4) especially established in the anti-parallel case could not be found by Gopalswamy et al. (2001). With respect to the density model, Gopalswamy et al. (2001) used a 10- and 3-fold Saito density model (Saito et al. 1977) for active and quiet regions, respectively, where the 3-fold Saito density model is similar to that used in this paper. The 3-fold Saito and one-fold Newkirk (1961) density model provide values of $5.08 \times 10^{8} \mathrm{~cm}^{-3}$ and $8.78 \times 10^{8} \mathrm{~cm}^{-3}$ at the base of the corona, respectively.

\section{Discussion}

In order to study the formation and development of shock waves and the associated type II bursts in the corona, a
Table 1. Parameters of shock formation in the case of parallelly $(+)$ and anti-parallelly (-) directed magnetic fields.

\begin{tabular}{lcccccc}
\hline \hline & \multicolumn{3}{c}{+} & & & - \\
\hline$\delta$ & $15^{\circ}$ & $45^{\circ}$ & $75^{\circ}$ & $15^{\circ}$ & $45^{\circ}$ & $75^{\circ}$ \\
\hline$V\left(\mathrm{~km} \mathrm{~s}^{-1}\right)$ & 750 & 910 & 2000 & 760 & 940 & 2000 \\
$R_{\mathrm{II}} / R_{\mathrm{S}}$ & 1.54 & 1.20 & 1.04 & 1.22 & 1.16 & 1.04 \\
$f_{\mathrm{pe}}^{*}(\mathrm{MHz})$ & 50 & 120 & 220 & 110 & 140 & 220 \\
\hline
\end{tabular}

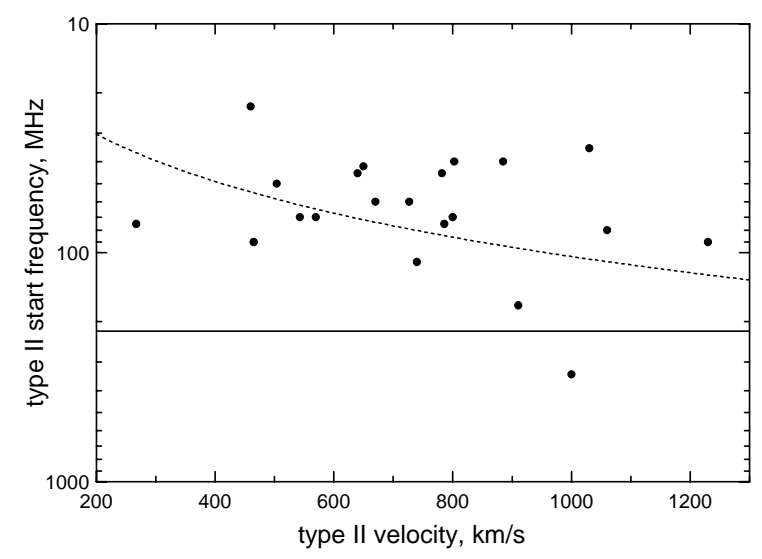

Fig. 7. Scatter plot of the start frequency of solar type II radio bursts versus its radial source velocity.

disturbance propagating obliquely away from an active region with a velocity $V$ is considered. Here, it is thought that it is travelling along a straight line making an angle $\delta$ with the $z$ axis (Fig. 3). Using the sample of solar type II radio bursts investigated by Klassen et al. (2000) the radial source velocities $V_{\mathrm{II}, \mathrm{r}}$ are in the range $267-1224 \mathrm{~km} \mathrm{~s}^{-1}$ with a mean value of $740 \mathrm{~km} \mathrm{~s}^{-1}$. The radial velocity $V_{\mathrm{r}}$ is related to the full velocity $V$ by $V_{\mathrm{r}}=V / \cos \alpha$, where $\alpha$ denotes the angle between the straight line and the radial direction (Fig. 3). Here, it is assumed that the Alfvén-Mach number of a shock should exceed (at least) 1.4 in order to accelerate electrons sufficiently for type II radio (i.e. "backbone") emission. Thus, the type II radio burst source is formed where $V_{\mathrm{r}} \cdot \cos \alpha=1.4 v_{\mathrm{A}}$ is fulfilled. Note that both $\alpha$ and $v_{\mathrm{A}}$ depend on $R$. The velocity $V$ of the disturbance and the radial distance $R_{\mathrm{II}}$, where the type II radio burst source is formed, can be determined by solving this relationship. That is done for several values of $\delta$ as summarized in Table 1. Here, the results of the radial behaviour of the Alfvén speed (Fig. 4) are adopted for these calculations and $V_{\mathrm{r}}=740 \mathrm{~km} \mathrm{~s}^{-1}$ (Klassen et al. 2000) is taken as a typical radial velocity of a type II burst source. In Table $1, f_{\mathrm{pe}}^{*}$ denotes the electron plasma frequency at the radial distance $R_{\mathrm{II}, \mathrm{f}}$. It is generally assumed that the type II radio radiation is generated by plasma emission (Melrose 1985). The emission takes place near the local electron plasma frequency $f_{\mathrm{pe}}=\left(e^{2} N_{\mathrm{e}} / \pi m_{\mathrm{e}}\right)^{1 / 2}$ ( $e$, elementary charge; $N_{\mathrm{e}}$, electron number density; $m_{\mathrm{e}}$, electron mass). In Fig. 7 the start frequency versus the radial velocity of type II radio burst sources is given for the sample of events considered by Klassen et al. (2000). The horizontal line represents $220 \mathrm{MHz}$. The start frequency of solar type II radio bursts should be below $220 \mathrm{MHz}$ according to Table 1. This is true for most of the cases in Fig. 7. Furthermore, the start 


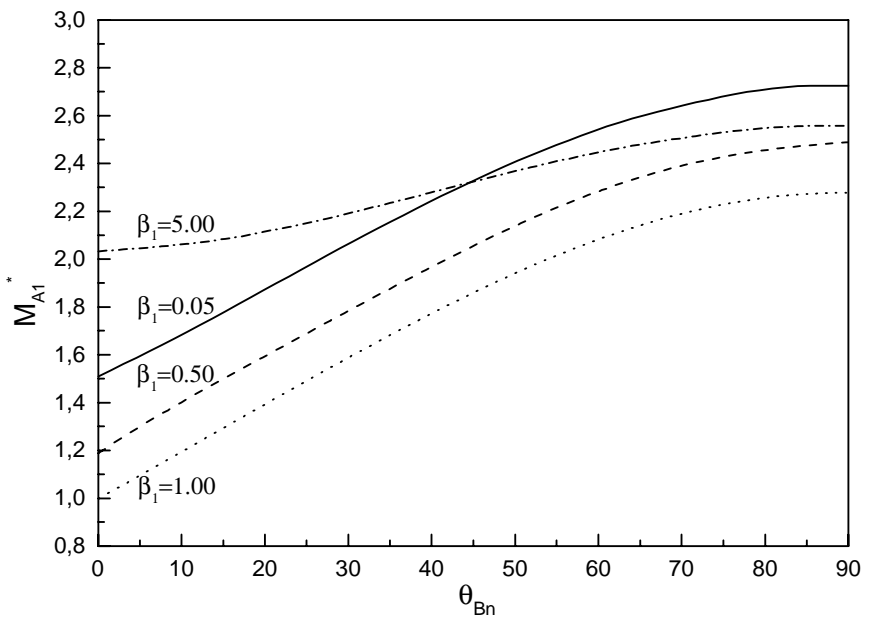

Fig. 8. Dependence of the critical Alfvén-Mach number on the angle $\theta_{\mathrm{Bn}}$ for several values of the plasma-beta.

frequency should increase with increasing velocity of type II burst sources according to the presented arguments. That is reflected in Fig. 7 by the dotted line, which represents the line of linear regression of the sample considered.

Now, the propagation of a disturbance travelling obliquely away from an active region in the low corona up to $1 \mathrm{AU}$ in the interplanetary space is considered. In order to estimate the typical spatial and temporal scales of this movement, the propagation of such a disturbance is thought to be along a straight line. Then, the velocity $V=925 \mathrm{~km} \mathrm{~s}^{-1}$ and the angle $\delta=45^{\circ}$ are chosen as typical (mean) values (Table 1). At the beginning it propagates into regions with decreasing Alfvén speeds according to Fig. 6, i.e. the Alfvén-Mach number is increasing. As already mentioned, the emission of solar type II radio bursts occurs if the Alfvén-Mach number exceeds roughly the value 1.4. Thus, the appearance of the type II burst would be expected at a distance of about $1.2 R_{\mathrm{S}}$. This distance will be reached 160-210 s after the initial energy release (Table 2). The further movement of the shock continues in regions of increasing Alfvén-Mach numbers whose maximum of 2.1 and 4.1 are reached at $1.45 R_{\mathrm{S}}$ and $1.32 R_{\mathrm{S}}$ for the parallel (+) and anti-parallel(-) case, respectively (Table 2). It is well-known that supercritical shock waves accelerate particles (Kennel et al. 1985). Figure 8 shows the dependence of the first critical Alfvén-Mach number $M_{\mathrm{A} 1}^{*}$ as defined by Edmiston et al. (1984) on the angle $\theta_{\mathrm{Bn}}$ between the upstream magnetic field and the shock normal for several values of the upstream plasma-beta. The plasma-beta is defined by the ratio of the kinetic pressure to the magnetic one, i.e. it is related to the Alfv́en- and sound speed by $\beta_{\text {plasma }}=8 \pi N k_{\mathrm{B}} T / B^{2}=2 c_{\mathrm{s}}^{2} / \gamma v_{\mathrm{A}}^{2}$. The plasma-beta has values of 0.2 and 0.8 at places where the Alfvén-Mach number takes its maximum in the parallel and anti-parallel case, respectively. Thus, a shock wave with an Alfvén-Mach number of 2.1 becomes supercritical in the case of a quasi-parallel shock geometry (i.e. $\theta_{\mathrm{B}}<45^{\circ}$ ) under coronal circumstances, whereas a shock with $M_{\mathrm{A}}=4.1$ is always supercritical (Fig. 8). Only in the anti-parallel case, the disturbance does become a strong supercritical shock, which accelerates both electrons and ions very efficiently. Consequently, such a shock wave can be a source of energetic particles outside active regions in the solar corona. That happens roughly 5 min after the initial energy release (Table 2). Such a temporal delay between the initial energy release and the production of energetic electrons has been reported by Krucker et al. (1999), Haggerty \& Roelof (2001), and Klassen et al. (2002). A similar phenomenon can also be seen in the solar type II radio burst on November 18, 2000 (Fig. 1). The SA type III bursts as the signature of energetic electron beams occurred roughly 3 min after the onset of the "backbone" radiation as the signature of the associated shock wave. Such a time delay could be caused by the occurrence of a minimum of the Alfvén speed (or maximum of Alfvén-Mach number) in the middle of the corona as discussed above. If the disturbance travels further outwards, it moves into regions of increasing Alfvén speed, where its Alfvén-Mach number decreases below 1.4, i.e. the associated solar type II radio burst would disappear. That happens at a distance of about $2.7 R_{\mathrm{S}}$ corresponding to an electron plasma frequency of $9 \mathrm{MHz}$ (Table 2). Thus, a solar type II burst would appear roughly $20 \mathrm{~min}$ in agreement with the observations. Furthermore, the same disturbance would be able to drive a supercritical shock wave (i.e. with $M_{\mathrm{A}}>1.5$ ) beyond a radial distance of about $6 R_{\mathrm{S}}$, which will be reached in about 1 hour after the inital energy release in the near-Sun interplanetary space.

Exact values of the scenario just discussed are given in Table 2. It summarizes the spatial and temporal scales of the formation and development of shock waves in the solar corona and near-Sun interplanetary space for the parallel (+) and antiparallel (-) case. The frequencies given in the 4 th and 7 th column represent the electron plasma frequencies expected at the radial levels of the 2 nd and 5 th column in Table 2. The travel time $t$ of a disturbance with the velocity $V$ from the origin of the straight line $\left(R=R_{\mathrm{S}} ; \rho=0\right)$ up to a point $P$ with a distance $R$ from the center of the Sun has been calculated by

$t=\frac{R_{\mathrm{S}}}{V} \cdot\left[\sqrt{\frac{R^{2}}{R_{\mathrm{S}}^{2}}-\sin ^{2} \delta}-\cos \delta\right]$

as given in the 3rd and 6th column in Table 2.

In the interplanetary space beyond $6 R_{\mathrm{S}}$ the shock geometry will be mainly quasi-parallel, i.e. $\theta_{\mathrm{Bn}}<45^{\circ}$, where the critical Alfvén-Mach number has values in the range 1.2-1.6 (Fig. 8). Consequently, most of the interplanetary shocks should be able to accelerate particles, which can be observed as so-called solar energetic particle events (SEPs) at 1 AU (Kahler 1994; Reames et al. 1996). Since SEPs are usually detected by spacecraft at $1 \mathrm{AU}$, the energetic particles must be transported from the acceleration site up to $1 \mathrm{AU}$. They are influenced by Coulomb collisions with the charged particles of the ambient plasma. The mean free path $\lambda_{\mathrm{mfp}}$ gives the length of the path of a particle with the velocity $v$ through a plasma with the full particle number density $N$ up to its deflection of more than $90^{\circ}$ from its initial direction. $\lambda_{\mathrm{mfp}}$ is depending on $v$ and $N$ according to $\lambda_{\mathrm{mfp}} \propto v^{4} / N$ (Krall \& Trivelpiece 1973). Therefore, on the one hand energetic electrons can more easily leave the dense corona than energetic ions because of their higher speeds. On the other hand, both energetic electrons and ions can travel from the 
Table 2. Typical spatial and temporal scales of the formation and development of shock waves.

\begin{tabular}{lcccccc}
\hline \hline direction of magnetic fields & \multicolumn{3}{c}{+} & & - & \\
\hline & $R / R_{\mathrm{S}}$ & $t(\mathrm{~s})$ & $f_{\mathrm{pe}}(\mathrm{MHz})$ & $R / R_{\mathrm{S}}$ & $t(\mathrm{~s})$ & $f_{\mathrm{pe}}(\mathrm{MHz})$ \\
\hline initial energy release & 1.00 & 0 & - & 1.00 & 0 & - \\
appearance of type II bursts & 1.20 & 210 & 120 & 1.16 & 160 & 140 \\
maximum of $M_{A}$ & 1.45 & 430 & 60 & 1.32 & 310 & 80 \\
disappearance of type II bursts & 2.51 & 1300 & 10 & 2.87 & 1600 & 7.4 \\
on-set of IP shock & 6.18 & 4200 & 1.7 & 5.58 & 3600 & 2.0 \\
\hline
\end{tabular}

acceleration site up to $1 \mathrm{AU}$ in the case of interplanetary shocks established beyond $6 R_{\mathrm{S}}$ because of the drastically lower particle number density in the interplanetary space.

\section{Conclusions}

The propagation of a disturbance away from an active region through the solar corona into the interplanetary space has been presented. Such a disturbance is initially produced by a flare or the lift off of a CME. Here, the magnetic field of an active region is modelled by a magnetic dipole. The complete magnetic field is a superposition of those of the magnetic dipole and the quiet Sun. Such a magnetic field structure leads to regions of a minimum of the Alfvén speed in the middle of the solar corona. The Alfvén speed takes a maximum of $740 \mathrm{~km} \mathrm{~s}^{-1}$ at a distance of $3.8 R_{\mathrm{S}}$ from the center of the Sun. The occurrence of such extrema has consequences for the development and formation of shock waves. A shock wave can be established at regions where the travelling disturbance becomes super-Alfvénic (i.e. $M_{\mathrm{A}}>1$ ). This is the case in the middle of the corona $\left(\approx 1.2-3 R_{\mathrm{S}}\right)$ and the near-Sun interplanetary space beyond $6 R_{\mathrm{S}}$. The occurrence of two shock waves, i.e. one in the corona and the other one in the interplanetary space, was actually observed during the solar event on May 12, 1997 (Gopalswamy \& Kaiser 2002).

The occurrence of a local maximum of the Alfvén speed in the near-Sun interplanetary space was already deduced by Mann et al. (1999a). Moreover, Gopalswamy et al. (2001) considered the radial behaviour of the fast magnetosonic speed above active regions, where they adopted the magnetic field model by Dulk \& McLean (1976), and already found a region of a minimum of the Alfvén speed in the middle of the corona. Thus, they concluded that there are two regions, i.e. in the middle of the corona and in the near-Sun interplanetary space, where shock waves can be established.

Shock waves can accelerate particles (electrons, protons and heavy ions) if they become supercritical (Kennel et al. 1985). That is the case in the corona in the range 1.2-2.9 $R_{\mathrm{S}}$ and near-Sun interplanetary space at distances beyond $6 R_{\mathrm{S}}$ from the center of the Sun. Especially in the case of antiparallel directed magnetic fields of the active region and of the quiet Sun, regions of low Alfvén speed occur in the corona. There, strong supercritical shock waves can be established and they become able to produce highly energetic particles. Energetic electrons are predominantly able to penetrate into the interplanetary space up to $1 \mathrm{AU}$ due to their high velocity and, consequently, large mean free path, in contrast to energetic ions.
Thus, the discussion provides a typical scenario of the formation of shock waves in the solar corona and the near-Sun interplanetary space and their association with solar energetic particle events:

- initial energy release (flare) and/or lift off of a CME;

- formation of a shock wave in the middle of the corona and the appearance of its associated type II burst;

- production of energetic electrons by the coronal shock wave;

- decay of the coronal shock wave in the upper corona;

- formation of a shock wave in the near-Sun interplanetary space beyond $6 R_{\mathrm{S}}$;

- production of energetic electrons and ions by the interplanetary shock observed as solar energetic particle events at $1 \mathrm{AU}$.

A similar scenario has already been discussed in a qualitative manner by Mann et al. (1999a) and Gopalswamy et al. (2001). Here, this study was generalized in a quantitative manner much more in detail. The different steps of events occur with a definite time delay, for which Table 2 gives typical values.

At the region of maximum Alfvén speed the plasma-beta is quite low, i.e. $\beta_{\text {plasma }}=0.1$. Then, the critical Alfvén-Mach number $M_{\mathrm{A}}^{*}$ is greater than 1.5. Consequently, only a disturbance with a velocity greater than $1100 \mathrm{~km} \mathrm{~s}^{-1}$ is able to travel as a supercritical shock wave through the corona into the interplanetary space (Mann et al. 1999a). Since CMEs have velocities with a mean value of about $400-500 \mathrm{~km} \mathrm{~s}^{-1}$ (St. Cyr et al. 2000), a part of them can drive a shock wave only in the middle of the corona and, later, in the interplanetary space due to the occurrence of the maximum of the Alfvén speed (Mann et al. 1999a; Gopalswamy et al. 2001).

Acknowledgements. This work was supported by the German Deutsche Forschungsgemeinschaft, DFG under project number MA 1376/14-1.

\section{References}

Aurass, H. 1996, in Coronal Physics from Radio and Space Observations, ed. G. Trottet, Lecture Notes in Physics (Heidelberg: Springer Verlag), 135

Aurass, H., Hofmann, A., \& Urbarz, H.-W. 1998, A\&A, 334, 289

Banaszkiewicz, M., Axford, W. I., \& McKenzie, J. F. 1998, A\&A, 337,940

Bougeret, J.-L., et al. 1995, in The Global Geospace Mission, ed. C. T. Russell (Dordrecht: Kluwer Academic Publishers), 263 
Cane, H. V., Stone, R. G., Fainberg, J. L., Stewart, R. T., \& Steinberg, J. L. 1981, Geophys. Res. Lett., 8, 1285

Cane, H. V. 1983, in Solar Wind Five, NASA Conf. Publ., CP-2280, 703

Classen, H. T., \& Aurass, H. 2002, A\&A, 384, 1098

Dulk, G. A., \& McLean, D. J. 1978, A\&A, 66, 315

Edmiston, J. P., \& Kennel, C. F. 1984, J. Plasma Phys., 32, 429

Gopalswamy, N., \& Kaiser, M. L. 2002, Adv. Space Res., 29, 307

Gopalswamy, N., Kundu, M. R., Manoharan, P. K., et al. 1997, ApJ, 486, 1036

Gopalswamy, N., Kaiser, M. L., Lepping, R. P., et al. 1998, J. Geophys. Res., 103, 307

Gopalswamy, N., Kaiser, M. L., Sato, J., \& Pick, M. 2000, in High Energy Solar Physics, ed. R. Ramaty, \& N. Mandzhavidze, PASP Conf. Ser., 206, 351

Gopalswamy, N., Lara, A., Kaiser, M. L., \& Bougeret, J.-L. 2001, J. Geophys. Res., 106, 25, 261

Haggerty, D. K., \& Roelof E. C. 2001, Proc. ICRC XXV (Copernicus Society), 3238

Kahler, S. W. 1994, ApJ, 428, 837

Kennel, C. F., Edmiston, J. P., \& Hada, T. 1985, in Collisionless Shocks in the Heliosphere: A Tutorial Review, ed. R. G. Stone, \& B. T. Tsurutani, Geophysical Monograph, 34 (Washington D.C.: AGU), 1

Klassen, A., Aurass, H., Klein, K.-L., Hofmann, A., \& Mann, G. 1999, A\&A, 343, 287

Klassen, A., Aurass, H., Mann, G., \& Thompson, B. J. 2000, A\&AS, 141,357

Klassen, A., Bothmer, V., Mann, G., et al. 2002, A\&A, 385, 1078

Klein, K.-L., Khan, J. I., Vilmer, N., Delouis, J.-M., \& Aurass, H. 1999, A\&A, 346, L53

Koutchmy, S. 1994, Adv. Space Res., 14, 29

Krall, N. A., \& Trivelpiece, A. W. 1973, Principles of Plasma Physics (New York: McGraw-Hill), 621

Krucker, S., Larson, D. E., Lin, R. P., \& Thompson, B. J. 1999, ApJ, 519,864

Landau, L. D., \& Lifschitz, E. M. 1975, The classical field theory (Oxford: Pergamon Press)

Leblanc, Y., Dulk, A., \& Bougeret, J.-L. 1998, Sol. Phys., 183, 165
Mann, G. 1995, in Coronal Magnetic Energy Release, ed. A. O. Benz, \& A. Krüger, Lecture Notes in Physics (Heidelberg: Springer Verlag), 183

Mann, G., Aurass, H., Voigt, W., \& Paschke, J. 1992, in Proc. First SOHO Workshop, ESA SP-348, 129

Mann, G., Aurass, H., Klassen, A., Estel, C., \& Thompson, B. J. 1999a, in Proc. 8th SOHO Workshop, ESA SP-446, 447

Mann, G., Jansen, F., MacDowall, R. J., Kaiser, M. L., \& Stone, R. G. 1999b, A\&A, 348, 614

Mariani, F., \& Neubauer, F. M. 1990, in Physics of the Inner Heliosphere, ed. R. Schwenn, \& E. Marsch (Heidelberg: Springer Verlag), 183

Melrose, D. 1985, in Solar Radio Physics, ed. D. J. McLean, \& N. R. Labrum (Cambridge: Cambridge Univ. Press), 177

Nelson, G. S., \& Melrose, D. 1985, in Solar Radio Physics, ed. D. J. McLean, \& N. R. Labrum (Cambridge: Cambridge Univ. Press), 333

Newkirk, G. A. 1961, ApJ, 133, 983

Parker, E. N. 1958, ApJ, 128, 664

Priest, E. R. 1982, Solar Magnetohydrodynamics (Dordrecht: Reidel)

Reames, D. V., Barbier, L. M., \& Ng, C. K. 1996, ApJ, 466, 473

Reiner, M. J., \& Kaiser, M. L. 1999, J. Geophys. Res., 104, 16, 979

Saito, K., Poland, A. I., \& Munro, R. H. 1977, Sol. Phys. 55, 121

Schwenn, R. 1990, in Physics of the Inner Heliosphere, ed. R. Schwenn, \& E. Marsch (Berlin, Heidelberg: Springer Verlag), 99

St. Cyr, O. C., Howard, R. A., Sheeley, N. R., et al. 2000, J. Geophys. Res., 105, 18169

Stewart, R. T., Howard, R. A., Hansen, F., Gergely, T., \& Kundu, M. R. 1974a, Sol. Phys., 36, 219

Stewart, R. T., McCabe, M., Koomen, M. J., Hansen, F., \& Dulk, G. 1974b, Sol. Phys., 26, 203

Suzuki, S., \& Dulk, G. A. 1985, in Solar Radio Physics, ed. D. J. McLean, \& N. R. Labrum (Cambridge: Cambridge Univ. Press), 289

Uchida, Y. 1960, PASJ, 12, 376

Uchida, Y., Altschuler, D., \& Newkirk, G. 1973, Sol. Phys., 28, 495

Vršnak, B., Ruždjak, V., \& Aurass, H. 1995, Sol. Phys., 158, 331

Wagner, W. J., \& MacQueen, E. M. 1983, A\&A, 120, 136

Wild, J. P., \& McCready, L. L. 1950, Austr. J. Sci. Res., Ser. A, 3, 387 